\title{
Effects of Multisensory Stimulation on a Sample of Institutionalized Elderly People With Dementia Diagnosis: A Controlled Longitudinal Trial
}

\author{
Ana Maseda, $\mathrm{PhD}^{1}$, Alba Sánchez, MD¹, Ma Pilar Marante, MD¹, Isabel González- \\ Abraldes, $\mathrm{PhD}^{1}$, Ana Buján, $\mathrm{PhD}^{1}$, and José Carlos Millán-Calenti, $\mathrm{PhD}^{1}$ \\ ${ }^{1}$ Department of Medicine, Faculty of Health Sciences, Gerontology Research Group, University of A Coruña, Campus de Oza, A \\ Coruña, Spain
}

\begin{abstract}
Long-term effects of multisensory stimulation were assessed using a "Snoezelen" room on older residents with dementia. Thirty patients were randomly assigned to 3 groups: multisensory stimulation environment (MSSE) group, individualized activities (activity) group, and control group. The MSSE and activity groups participated in two 30minute weekly individualized intervention sessions over 16 weeks. Pre-, mid-, posttrial, and 8-week follow-up behavior, mood, cognitive, and functional impairment in basic activities of daily living were registered. Items included in the physically nonaggressive behavior factor improved significantly in post- versus pretrial in the MSSE group compared to the activity group, with no significant differences between MSSE and control groups. The MSSE and activity groups demonstrated behavior improvements and higher scores on the Cohen-Mansfield agitation inventory, verbal agitated behavior factor, and Neuropsychiatric Inventory-Nursing Home, with no significant differences between groups. The MSSE could have long-term positive effects on such neuropsychiatric symptoms in older people with dementia.
\end{abstract}

Keywords: multisensory stimulation, dementia, elderly, agitation

\section{Introduction}

The multisensory stimulation environment (MSSE) was developed in the Netherlands in the 1970s and was first introduced to people with learning difficulties. ${ }^{1}$ Since the beginning of the 1990s, the MSSE has been used as a nonpharmacological therapy for people with dementia. ${ }^{2}$ The multisensory stimulation typically occurs in a specifically designed room known as a multisensory stimulation room (MSSR). This room includes many objects that pertain to the 5 senses, including fiber-optic cables, water columns, aroma therapy, different music/sounds, tactile objects, and screen projectors among others. ${ }^{3}$

Elderly people with dementia, particularly those who are institutionalized, are exposed to either sensory deprivation or excessive sensory stimulation. The imbalances in the pacing of sensory-stimulation or sensory-calming activity affect the behavior and the instrumental and social function of institutionalized people with dementia. ${ }^{4}$ The Mini-Mental State Examination (MMSE) might contribute to overcome these problems since it provides a stress-free, entertaining environment for both stimulation and relaxation. ${ }^{5}$

One of the distinguishing elements of MSSE compared to other therapies is the one-to-one attention and the adoption of a nondirective approach, which encourages patients to engage with sensory stimuli of their choice. ${ }^{6}$ Because MSSE does not appeal to cognitive abilities, it is one of the few approaches that are suitable for persons with severe or very severe dementia and limited verbal communication capabilities. ${ }^{7}$ Nonpharmacological interventions are recommended as first-line therapies for patients with these characteristics due to the safety concerns related to pharmacologic therapies. ${ }^{8}$

In a recent revision, ${ }^{9}$ we found that MSSR stimulation had immediate positive effects on the behavior and mood10-14 of people with dementia. However, there are no conclusive data on the long-term effectiveness, that is, the maintenance of the positive effects outside of the session time and place, of the MSSE. 


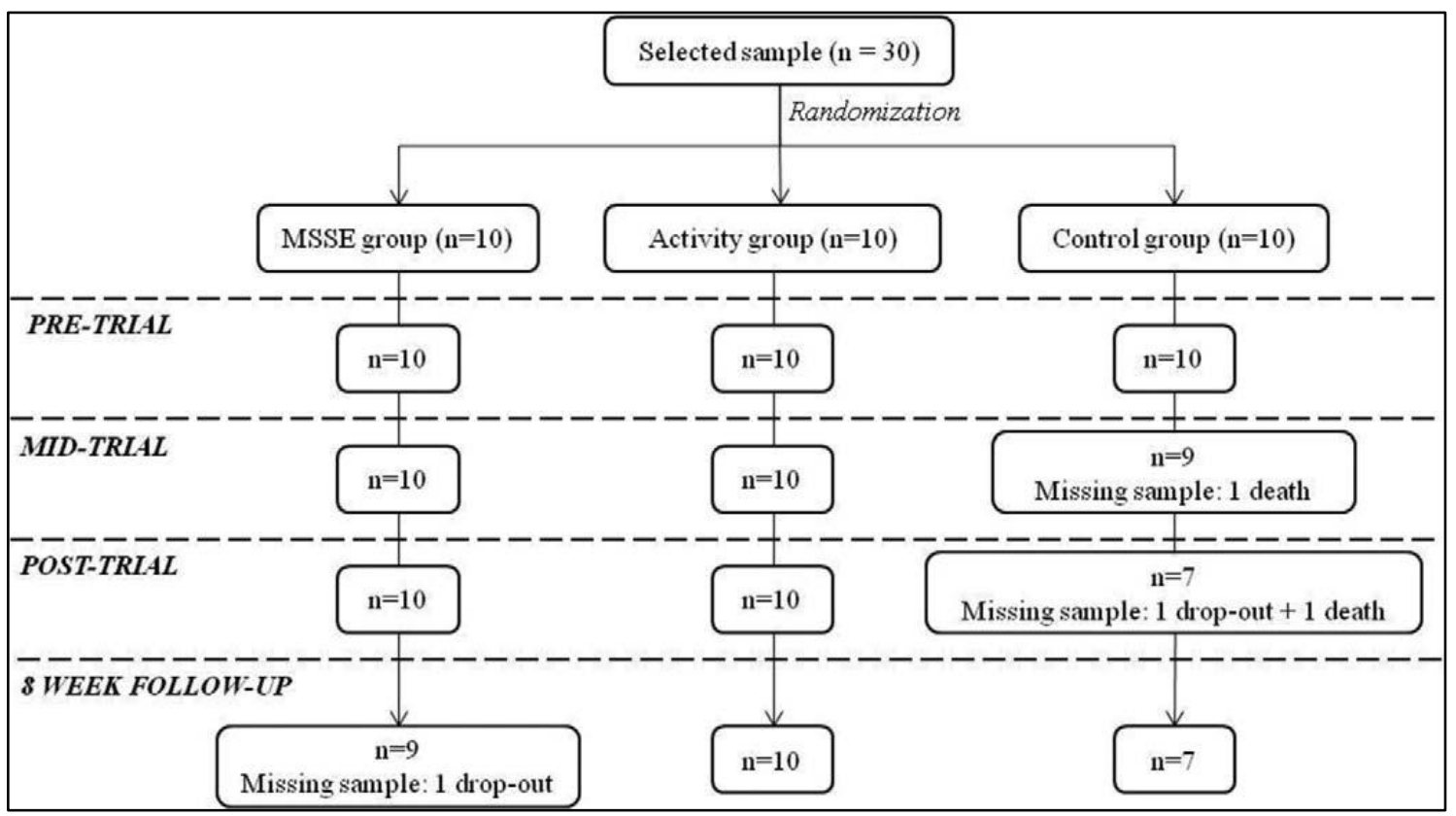

Figure 1. Consolidated Standards of Reporting Trial (CONSORT) diagram. MSSE indicates multisensory stimulation environment.

Most of the articles published on the use of the MSSE with people with dementia have mainly focused on its effects on behavior and mood. There are very few data on the long term effectiveness of the MSSE in relation to patient's cognitive status ${ }^{15}$ and functional status. ${ }^{16,17}$

There is no evidence demonstrating a higher effectiveness in MSSE than in one-to-one activities that were frequently used in patients with dementia, and with a clear aim and focus, for example, playing cards, looking at photographs, playing games, or doing puzzles. ${ }^{6,15}$ Therefore, more studies including oneto-one control groups are necessary to be able to distinguish the beneficial effects of MSSE from those of one-toone activity.

Therefore, the main objective of the current study was to evaluate the long-term effectiveness of the MSSE in regard to the behavior, mood, and cognitive and functional impairment in basic activities of daily living (ADLs) of institutionalized elderly patients with dementia.

\section{Methods}

Design

We conducted a controlled longitudinal study, where participants were stratified according to their cognitive and functional status onADLs, and later, randomly assigned to 3 groups (MSSE, activity, and control).

\section{Patients}

The sample was selected among the residents of a specialized elderly center in A Corunña (Spain).

Dementia diagnosis was noted on the medical history and provided by a neurologist before placement in the gerontological complex, being corroborated by the elderly center's medical doctor. Global Deterioration Scale (GDS) was applied by a clinical psychologist with experience in assessing people with dementia to determine disease severity: mild, moderate, or severe dementia scored from levels 4 to 7. The exclusion criterion was the presence of sensory disorders that did not allow interaction with the multisensory stimulation objects.

A clinical psychologist checked the eligibility of the participants according to the inclusion/exclusion criteria, and the final sample consisted of 30 patients. A computer-based random number generator was used to randomly divide the sample into 3 groups of 10 participants. The patients' progress through the trial is shown in a Consolidated Standards of Reporting Trial (CONSORT) diagram (Figure 1). 
The study protocol was approved by the ethics committee at the University of A Coruña (Spain). Before the data collection, all participants were informed about the study and signed the corresponding informed consent. A proxy was used as a legally authorized representative to provide informed consent for elderly people with dementia to participate in research.

\section{Procedure}

The MSSE group participated in multisensory sessions in an MSSR that included various elements to stimulate the senses, including fiber-optic cables, water columns, a vibrating water bed, a mirror ball, screen projectors, video, an interactive projecting system, musical selections, aroma therapy equipment, a

tactile board with various textures, and so on.

The activity group participated in a series of one-to-one activity sessions, in which intellectual and/or physical demands were placed on the individual, and the approach was directive. These participants were asked to take part in activities such as playing cards, quizzes, or viewing photographs. ${ }^{15}$ This group was included in the study to differentiate the specific benefits of the multisensory stimulation from those derived from attending one-to-one therapy sessions. ${ }^{6}$

Finally, the control group did not participate in any of the above-mentioned activities; rather, this group continued with the daily routines of the center, including cognitive stimulation group sessionsconsisting of themed activities to orientate and actively stimulate cognition (focused on patients with GDS 4-6); training on ADL-which consists of guided performance providing the minimal required assistance to completely target ADLs (GDS 4-6); education and training of nursing assistants in dementia knowledge; acknowledgment of resident's experiences; and communication techniques and behavior management (GDS 4-7).

The design of the sessions followed by the MSSE and activity groups was based on the protocol suggested by Baker et $\mathrm{l}^{18}$ (Table 1). Nonspecific variables such as the therapist-patient ratio (1:1) and the number, frequency, and length of sessions were equivalent for the MSSE and activity groups. All patients from both groups took part in 2 weekly sessions for 16 weeks. These sessions lasted 30 minutes, unless the participant expressed a desire to leave. Sessions in both groups followed an internal structure that involved an introduction to the session, carrying the session through, and winding the session down. Nevertheless, in the MSSE group, there was flexibility within the standardization, in keeping with the traditional philosophy of the multisensory stimulation.

Table 1. Similarities and Differences Between MSSE and Activity Sessions. ${ }^{a}$

\begin{tabular}{lll}
\hline & MSSE Group & Activity Group \\
\hline Therapist approach & Nondirective & Directive \\
Multisensory stimulation & Efforts to stimulate all senses except taste & Nonintentional multisensory experience \\
Nature of stimuli & Unpatterned stimuli, nonsequential stimuli & Patterned, often sequential stimuli \\
Demands made on patient & No intellectual/physical demands & Intellectual/physical demands \\
Number of sessions & 32 & 32 \\
Frequency of sessions & Twice weekly & Twice weekly \\
Length of session & 30 Minutes & 30 Minutes \\
Location of sessions & MSSR & Quiet room away from others \\
Interaction with the therapist & One-to-one attention & One-to-one attention \\
Therapist profession & Psychologist and occupational therapist & Psychologist and occupational therapist \\
Therapist training & MSSE training and experience & Activity training and experience \\
Timing of measurement & Pre-, mid-, post-, and follow-up & Pre-, mid-, post-, and follow-up \\
& & \\
\hline
\end{tabular}

Abbreviations: MSSE, multisensory stimulation environment; MSSR, multisensory stimulation room.

${ }^{a}$ Adapted from Baker et al

All sessions were conducted by professionals (occupational therapist or psychologist) with equivalent education and training in the methodology used. To avoid the creation of positive or negative expectations, the MSSE and the activity sessions were presented to the staff and caregivers as 2 equally valid therapies.

As a result of this design, the differences found between the 2 conditions could be specifically attributed to the multisensory stimulation rather than more general therapeutic effects, such as the one-toone attention to the patients. 
The difference between the 2 types of intervention was caused by the characteristics that define the MSSE. The MSSE group used multisensory unpatterned stimuli, the therapist followed a nondirective approach, and the therapy required little intellectual or physical demands that are suitable for people with severe or very severe dementia and with limited verbal and psychomotor capabilities. By contrast, during the activity sessions, no special intentional multisensory experiences were introduced, the therapist followed a directive approach, and intellectual and/or physical demands were placed on the individual.

Data on the participants' sensorial preferences and interests were previously collected to design the content of the sessions and minimize the behavioral problems that some participants could present within the MSSE and activity contexts. In the MSSE group, sensorial preferences in the MSSR were assessed based on the procedure suggested by Pace et al. ${ }^{19}$ Furthermore, relatives of the participants of both groups were interviewed, with the aim of identifying the participants' hobbies, interests, and tastes.

All patients were assessed before the intervention (pretrial, week 0), in the middle of the intervention (mid-trial, week 8), at the end of the intervention (posttrial, week 16), and at 8 weeks after completing the intervention (8-week follow-up) for long-term monitoring of their behavioral, emotional, cognitive, and functional levels on ADLs. The period of long-term assessments has been established in the MSSE context 1 month after sessions. ${ }^{15}$

\section{Assessment Instruments}

The validated Spanish version ${ }^{20}$ of the Cohen-Mansfield Agitation Inventory (CMAI) ${ }^{21}$ was used to assess the frequency of manifestations of agitated behaviors. The CMAI consists of 30 items that are each rated on the following 7-point scale of frequency: $1=$ never; $2=$ less than once a week but still occurring; $3=$ once or twice a week; $4=$ several times a week; $5=$ once or twice a day; $6=$ several times a day; and 7 = several times an hour. The total score is obtained by summing the scores of each of the items. Utilizing factor analysis, Cohen-Mansfield et $\mathrm{al}^{21}$ found that the following 3 meaningful dimensions of agitation emerged in the nursing home: "aggressive behavior" (hitting, kicking, pushing, scratching, tearing things, cursing or verbal aggression, and grabbing); "physically nonaggressive behavior" (pacing, inappropriate robing or disrobing, trying to get to a different place, handling things inappropriately, general restlessness, and repetitious mannerisms); and "verbally agitated behavior" (complaining, constant requests for attention, negativism, repetitious sentences or questions, and screaming). In the current study, for each factor the total score was obtained by summing the scores of the corresponding items.

Behavior was assessed using the Spanish version ${ }^{22}$ of the Neuropsychiatric Inventory-Nursing Home (NPI-NH). ${ }^{23}$ The NPI-NH is a screening tool that was developed to characterize the neuropsychiatric disorders of institutionalized patients with dementia. For this tool, information is gathered from professional caregivers. The NPI-NH includes the following 12 neuropsychiatric symptoms: delusions, hallucinations, agitation/aggression, depression, anxiety, euphoria, apathy/indifference, disinhibition, irritability/lability, aberrant motor behavior, sleep and nighttime behavior disorders, and appetite and eating disorders. These symptoms are rated on a Likert-type scale according to their frequency (1-4), severity (1-3), and occupational disruptiveness (0-5). Two independent final scores, a total score and an occupational disruptiveness score, are obtained. For the total score, the frequency and severity of each specific symptom are multiplied; then, the values for all of the symptoms are summed. This score ranges from 0 (no evidence of neuropsychiatric disorders) to 120 when it is calculated from the scores obtained in the first 10 domains. When the neurovegetative symptoms are of significant importance, the score extends to 144 and is calculated from the scores obtained in the 12 domains. The final score of the occupational disruptiveness scale is obtained by summing the scores of occupational disruptiveness of each domain. This score ranges from 0 to 50, reaching 60 points if the last 2 domains are included.

The Cornell Scale for Depression in Dementia (CSDD) ${ }^{24}$ was used to assess mood. It is a tool that was specifically developed to assess signs and symptoms of major depression in patients with dementia. Information is elicited through 2 semistructured interviews; an interview with an informant and an interview with the patient. The CSDD consists of 19 items that are classified on a severity scale that ranges from 0 to $2(0=$ absent, $1=$ mild or intermittent, and $2=$ severe $)$. Total score is obtained by summing the scores for all items. Scores $>10$ indicate probable major depression. Scores $>18$ indicate definite major depression.

The participants' cognitive status was assessed using the $\mathrm{MMSE}^{25}$ adapted for the Spanish population. ${ }^{26}$ This questionnaire includes items that assess 5 cognitive domains. The maximum score of 30 corresponds to the highest cognitive status. The cutoff score for the presence of cognitive impairment is 24/25 (non cognitive impairment above 24). In the current study, the MMSE scores were adjusted by age and educational level. 
The $\operatorname{GDS}^{27}$ was used to measure the severity of dementia. This scale divides the progression of dementia into 7 stages of ability, from GDS 1 (no cognitive decline) to GDS 7 (very severe cognitive decline).

The patients' functional status, defined as the capacity to carry out basic everyday tasks, was assessed by an occupational therapist using the Barthel index ${ }^{28}$ validated for Spanish population. ${ }^{29}$ This index measures the following 10 ADLs: feeding, bathing, dressing, grooming, bowels, bladder, toilet use, transfers, mobility, and stairs. The item scores are summed to create a total score.

Scores range from 0 to 100 (90 in case the patient uses a wheelchair). The cutoff scores are as follows: $<20$, total dependence; 20 to 35 , severe dependence; 40 to 55 , moderate dependence; and >=60, mild dependence. $^{29}$

\section{Statistical Analysis}

Sample characteristics were summarized as frequency and percentage for the categorical variables and as mean and standard deviation (SD) for the continuous variables. The Student $\mathrm{t}$ test for continuous variables and the chi-square test for categorical variables were used to examine the differences in sociodemographic characteristics between the 3 groups at baseline. Finally, repeated measures analysis of variance (ANOVA) was used to assess performance differences in behavior, mood, and cognitive and functional status in terms of ADL over the pre-, mid-, and posttrial assessment points. In the first analysis, the within-subject variable was the measures over time (pre-, mid-, and posttrial assessment) and the between-subject variable included the group (MSSE and activity). In the second analysis, the withinsubject variable was the measures overtime (pre-, mid-, and posttrial assessment) and the between-subject variable included the group (MSSE and control).

In addition, repeated measures ANOVA was used to assess performance differences in behavior, mood, and cognitive and functional status in ADL between the posttrial and the 8-week follow-up. In this case, in the first analysis, the within-subject variable was the measures over time (posttrial assessment and follow-up) and the between-subject variable included the group (MSSE and activity). In the second analysis, the within-subject variable was the measures over time (posttrial assessment and follow-up) and the between-subject variable included the group (MSSE and control).

Differences between groups were tested by a group-time interaction. Statistical significance was set at a $\mathrm{P}$ value of less than .05. Statistical analysis was performed using the SPSS (SPSS Inc, Chicago, Illinois) version 18.30

\section{Results}

Table 2 shows the sociodemographic characteristics of the sample at baseline. The mean age of the sample was 87.3 years $(\mathrm{SD}+5.3$ ). Of the participants, $90.0 \%$ were women. Concerning marital status, $70 \%$ of the patients were widowed and $23.3 \%$ were single. Furthermore, $16.7 \%$ had no formal education and $33.3 \%$ had only primary education. We found no significant differences between the groups in age, gender, marital status, or educational level.

\section{Effect on Behavior}

With regard to agitation, as assessed by the CMAI, when comparing the MSSE group and the activity group, we observed an improvement in the physically nonaggressive behavior scores in the MSSE group in comparison with the activity group $\left(\mathrm{F}_{2}, 36=4.172, \mathrm{P}=.023\right)$ between pre-, mid-, and postintervention (Figure 2). Furthermore, there were significant time effects in both groups in the CMAI total score $\left(\mathrm{F}_{2,36}\right.$ $=3.281, \mathrm{P}<.049)$ and verbally agitated behavior $\left(\mathrm{F}_{2,36}=10.540, \mathrm{P}<.001\right)$, with an improvement in the level of agitation during the intervention. However, no significant differences were found between the groups (Figure 2). With regard to the CMAI aggressive behavior factor, no significant intervention effects or intergroup differences were found between pre-, mid-, and postintervention assessments.

When comparing the MSSE group and the control group, a significant decrease in verbally agitated behavior was observed from the pretrial to the posttrial in both groups $\left(\mathrm{F}_{2,30}=5.159, \mathrm{P}=.012\right)$, with no significant intergroup differences (Figure 2). With regard to the CMAI total score, the aggressive behavior factor, and the physically nonaggressive behavior factor, no significant intervention effects or intergroup differences between pre-, mid-, and postintervention assessments were found. When comparing postintervention and follow-up, no significant time effects or intergroup differences were observed in any of the CMAI scores. 
Table 2. Sociodemographic Characteristics of the Residents With Dementia at Week 0 (Baseline, Pretrial). ${ }^{\mathrm{a}}$

\begin{tabular}{|c|c|c|c|c|c|c|c|}
\hline & $\begin{array}{l}\text { MSSE } \\
(\mathrm{n}=10)\end{array}$ & $\begin{array}{c}\text { ACT } \\
(n=10)\end{array}$ & $\begin{array}{l}\text { Control } \\
(\mathrm{n}=10)\end{array}$ & $\begin{array}{c}\text { Total } \\
(\mathrm{n}=30)\end{array}$ & $\begin{array}{c}\text { P Value } \\
(\mathrm{MSSE}-\mathrm{ACT})\end{array}$ & $\begin{array}{c}\text { P Value } \\
\text { (MSSE-Control) }\end{array}$ & $\begin{array}{c}\text { P Value } \\
\text { (ACT-Control) }\end{array}$ \\
\hline \multicolumn{8}{|l|}{ Age, years } \\
\hline Mean (SD) & $87.2(6.8)$ & $87.9(4.7)$ & $86.7(4.5)$ & $87.3(5.3)$ & .793 & .568 & .850 \\
\hline Minimum-maximum & $77-96$ & $79-94$ & $79-92$ & $77-96$ & & & \\
\hline \multicolumn{8}{|l|}{ Gender, n $(\%)$} \\
\hline Female & $10(100.0)$ & $9(90.0)$ & $8(80.0)$ & $27(90.0)$ & .305 & .136 & .531 \\
\hline Male & $0(0.0)$ & $1(10.0)$ & $2(20.0)$ & $3(10.0)$ & & & \\
\hline \multicolumn{8}{|l|}{ Marital status, n (\%) } \\
\hline Single & $3(30.0)$ & $1(10.0)$ & $3(30.0)$ & $7(23.3)$ & .264 & .311 & .126 \\
\hline Married or partner & $0(0.0)$ & $0(0.0)$ & $2(20.0)$ & $2(6.7)$ & & & \\
\hline Widowed & $7(70.0)$ & $9(90.0)$ & $5(50.0)$ & $21(70.0)$ & & & \\
\hline Separated or divorced & $0(0.0)$ & $0(0.0)$ & $0(0.0)$ & $0(0.0)$ & & & \\
\hline \multicolumn{8}{|l|}{ Educational level, n (\%) } \\
\hline No formal education & $2(20.0)$ & $2(20.0)$ & $1(10.0)$ & $5(16.7)$ & .605 & .117 & .771 \\
\hline Primary & $2(20.0)$ & $3(30.0)$ & $5(50.0)$ & $10(33.3)$ & & & \\
\hline Secondary & $6(60.0)$ & $3(30.0)$ & $1(10.0)$ & $10(33.3)$ & & & \\
\hline College or higher degree & $0(0.0)$ & $2(20.0)$ & $3(30.0)$ & $5(16.7)$ & & & \\
\hline
\end{tabular}

Abbreviations: MSSE, multisensory stimulation environment; ACT, activity; SD, standard deviation. a Significance: $P$ value $<.05$.

Significant time effects were also found in the NPI-NH (Figure 3) when comparing the total punctuations in the MSSE group and in the activity group. Comparing the scores, both groups reflected a significant improvement in behavior $\left(\mathrm{F}_{2,36}=4.513, \mathrm{P}=.018\right)$, although the differences between the 2 groups were not significant. In both cases, the positive effects of the intervention did not continue in the follow-up period.

There was a significant increase in scores in the posttrial $\left(\mathrm{F}_{1,17}=6.737, \mathrm{P}=.019\right)$; thus, patients displayed worse behavior. When comparing the MSSE group with the control group, a significant increase in the NPI-NH total punctuation was found in both groups in the follow-up period $\left(\mathrm{F}_{1,14}=5.601\right.$, $\mathrm{P}=.033$ ), although no significant differences were found between the groups.

Comparing the MSSE group with the control group, significant time effects $\left(\mathrm{F}_{2,30}=3.575, \mathrm{P}=.040\right)$ were observed for the NPI-NH (Figure 3) occupational disruptiveness scale among pre-, mid-, and postintervention results, with a decrease in the scores for these groups.

However, there was an increase in the scores of both groups in the follow-up period $\left(\mathrm{F}_{1,14}=5.151, \mathrm{P}=\right.$ .040), with no significant differences between the groups.

\section{Effect on Mood}

With regard to mood, both the MSSE group and the activity group displayed improvement in the CSDD scores during intervention, although the results were not statistically significant. For both groups, the scores worsened in the follow-up period compared to the posttrial assessment $\left(\mathrm{F}_{1,17}=6.166, \mathrm{P}=.024\right)$, with no significant differences between the groups (Figure 4).

\section{Effect on Cognitive Level}

The MMSE scores remained stable during the intervention period in the 3 study groups (Figure 5). When comparing the MSSE group and the control group, a significant time effect was observed, with an important decrease in the scores of both groups between the posttrial assessment and the follow-up period $\left(\mathrm{F}_{1,12}=5.457, \mathrm{P}=.038\right)$. No significant differences were found between the 2 groups. For the GDS (Figure 5), there was a slight decrease in the MSSE group scores between pre-, mid-, and postintervention assessments that was not observed in the other 2 groups, but the results were not statistically significant.

\section{Effect on Functional Status in ADL}

There was an improvement in the Barthel index scores between pre-, mid-, and postintervention assessments in the MSSE group but not in the activity group or the control group (Figure 6). Between the posttrial assessment and the followup period, there was an important decrease in the MSSE group scores, achieving almost the baseline values.

However, the results were not significant. 


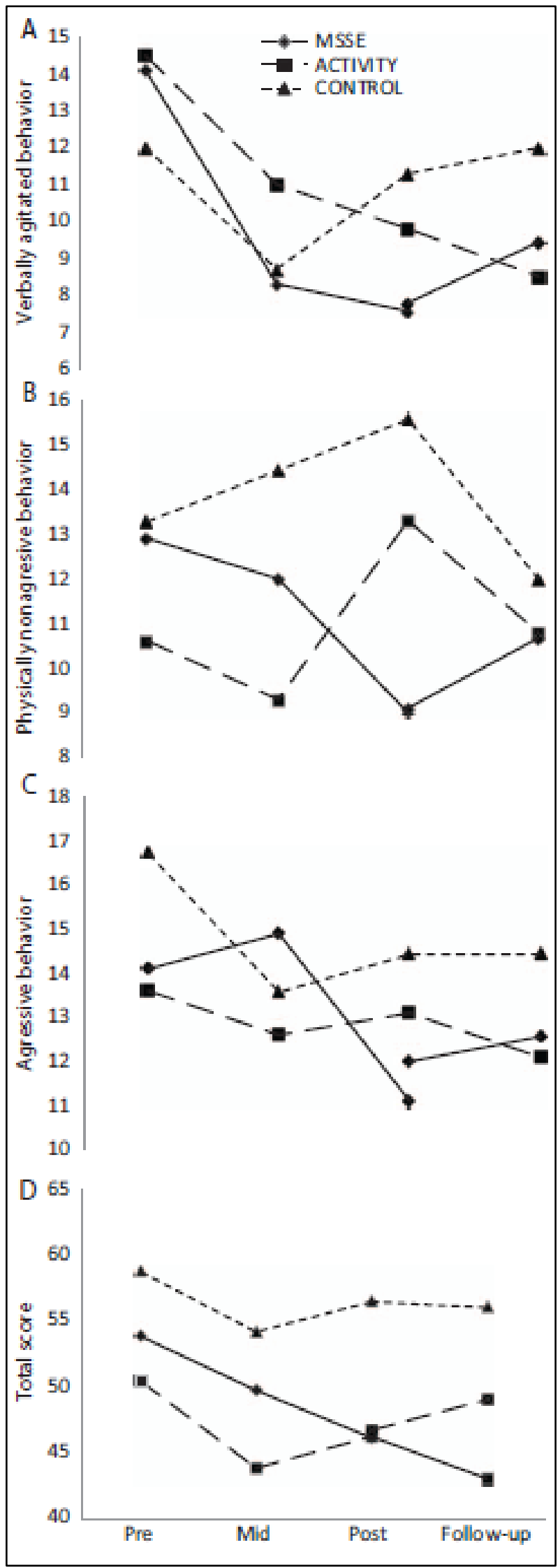

Figure 2. Cohen-Mansfield Agitation Inventory (CMAI) - physically nonaggressive behavior scores (A), verbally agitated behavior scores (B), aggressive behavior (C), and total scores (D) during the trial and follow-up (higher scores $1 / 4$ worse behavior). 


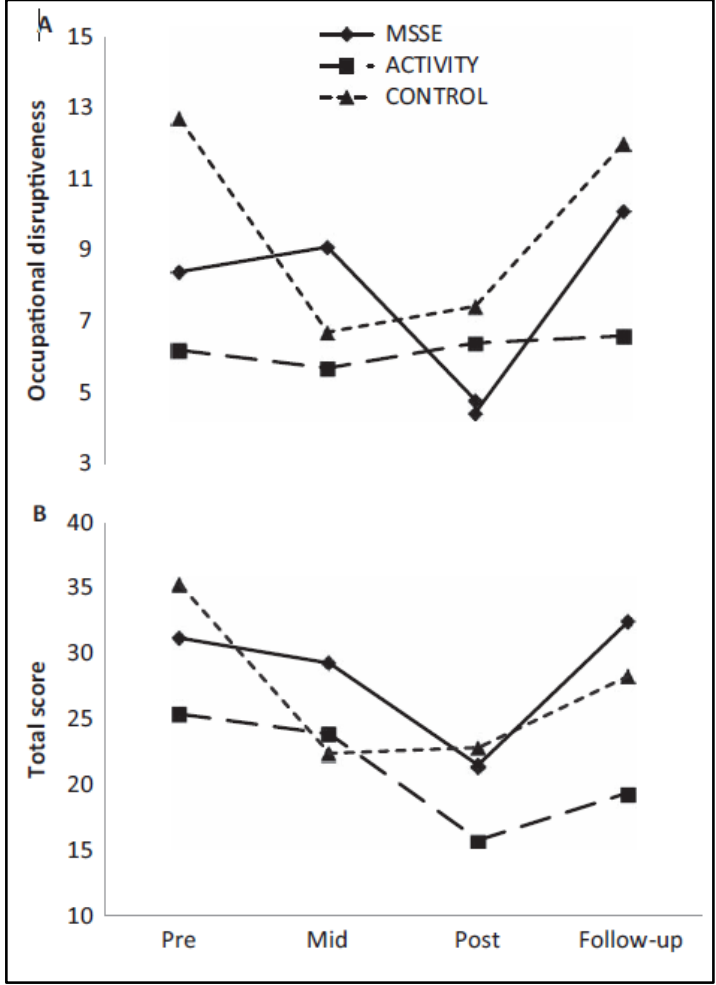

Figure 3. Neuropsychiatric Inventory-Nursing Home (NPI$\mathrm{NH}$ - occupational disruptiveness scores (A) and total scores (B) during the trial and follow-up (higher scores 1/4 worse behavior

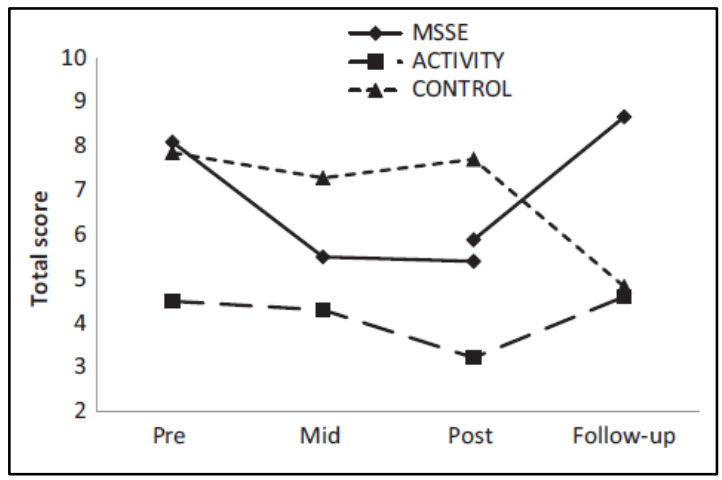

Figure 4. The Cornell Scale for Depression in Dementia (CSDD) - total scores during the trial and follow-up (higher scores $=$ worse mood) .

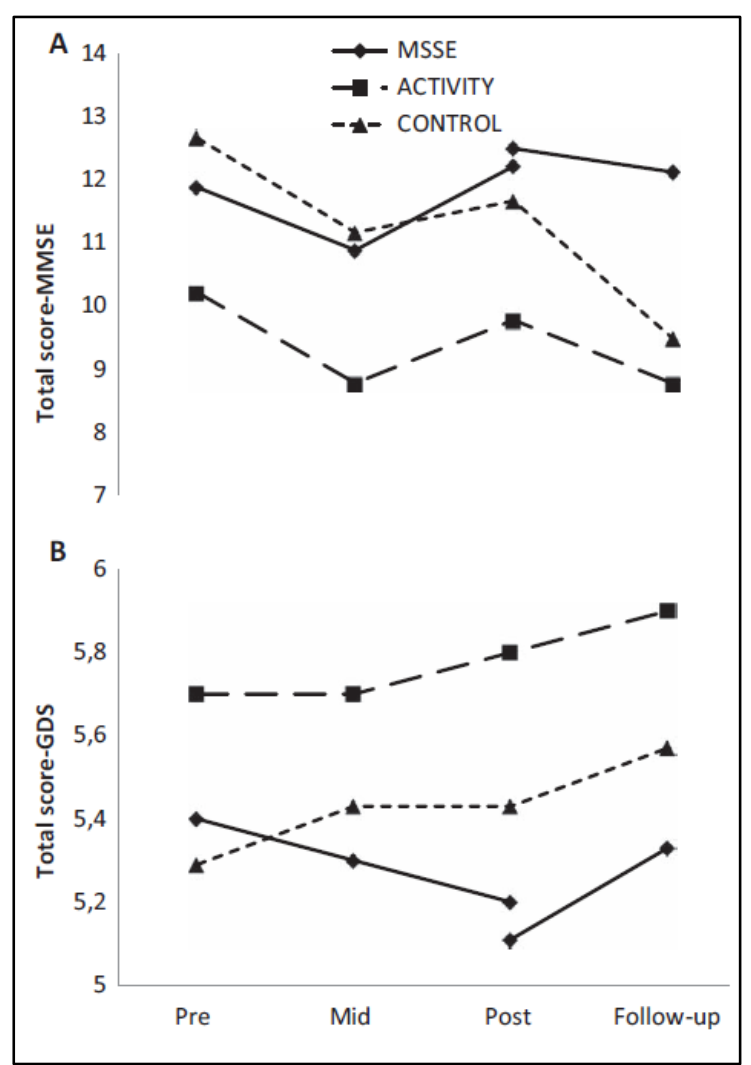

Figure 5. Cognitive scales: Mini-Mental State Examination (MMSE, lower scores = worse cognitive state; A) and Global Deterioration Scale (GDS, higher scores = worse cognitive state; B). Total scores during the trial and follow-up.

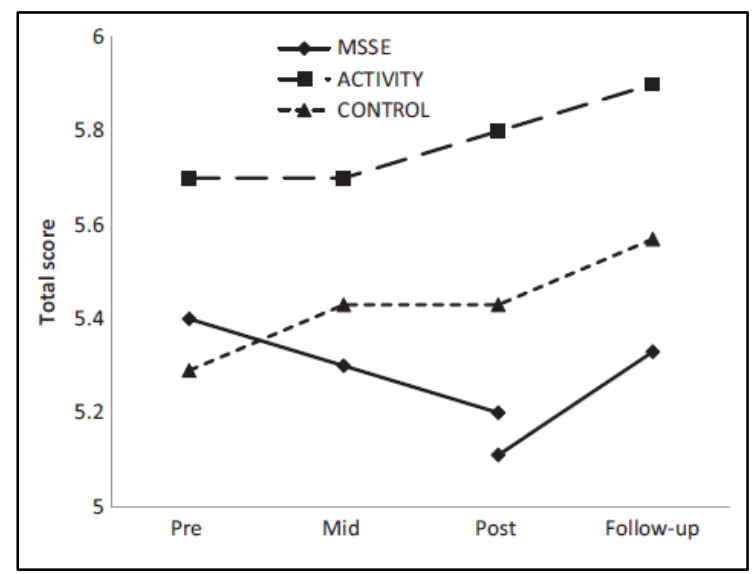

Figure 6. The Barthel index - total scores during the trial and followup (lower scores $=$ higher dependence level).

\section{Discussion}

\section{Effect on Behavior}

According to the current study, the main long-term benefits of MSSE are observed in behavioral problems, particularly agitated behavior. Improvement in the MSSE group was significantly higher than that in the activity group for one of the CMAI factors, physically nonaggressive behavior. On the other hand, both groups showed improvements in the CMAI total score and verbally agitated behavior factor, 
with no significant differences between the groups. Likewise, there was an improvement in the NPI-NH total punctuation in both groups (MSSE and activity group), with no significant differences between the groups. One explanation for this lack of differences could be that the one-to-one interaction with the therapist improved the patients' behavior rather than the multisensory stimulation.

For most people with dementia, neuropsychiatric symptoms occur during the course of the illness. These symptoms typically cause distress to the patients and caregivers and require intervention. ${ }^{31,32}$ Practical guidelines for handling neuropsychiatric symptoms recommend nonpharmacological interventions as a first therapy in these cases. ${ }^{31,33}$ Previous studies have provided evidence that MSSE produces immediate positive effects on agitation and other neuropsychiatric symptoms of dementia. ${ }^{9}$ However, in assessing this intervention, evaluation of long-term effectiveness is more acceptable and relevant to practice. ${ }^{34}$ Few studies have analyzed the long-term effects of this multisensory stimulation in MSSRs compared with an adequate control condition. In general, the observed effects are discreet, and the main long-term benefits are, as in the current case, evident in the patients' behavioral symptomatology. ${ }^{3,15,16}$ Baker et $\mathrm{al}^{15}$ studied the effects of an MSSR intervention in comparison with a control group that followed one-to-one activity sessions (playing cards, viewing photographs, or taking quizzes). After 4 weeks of intervention, both groups showed behavioral improvements in the "active/disturbed" subscale of the Behavior and Mood Disturbance scale (BMD; P < .05). No significant differences were found between the groups.

Milev et al $^{3}$ carried out a 24-week study (12-week intervention and 12-week follow-up) that observed a significant improvement in the behavior of the patients who received 1 to 3 weekly sessions in an MSSR compared to a control group that followed the center's daily routine. This difference was observed using the Daily Observation Scale in week $8(\mathrm{P}=.04)$ and the Clinical Global Impression-Improvement in week $12(\mathrm{P}=.05)$.

Patients with Alzheimer's disease16 who received sporadic MSSR sessions showed a decreased number of psychotic behaviors after a 3-month intervention compared to patients who continued with their daily routine.

With regard to the persistence of positive effects following the intervention, we did not find significant differences in the CMAI total punctuation, physically nonaggressive behavior, or verbally agitated behavior score between the posttrial and the follow-up period. However, the improvements observed in the NPI-NH total punctuation during the intervention were lost in the follow-up period. Previous results on the maintenance of the positive effects of the MSSE after intervention completion are not conclusive.

Milev et $\mathrm{al}^{3}$ found that the improvement in the behavior remained even 12 weeks after intervention, whereas Baker et $\mathrm{al}^{15}$ found that the improvement observed during the intervention period was lost 1 month later.

From the studies carried out for the time being, we cannot conclude positive effects of MSSE on behavior of people with dementia beyond those derived from attending one-to-one therapy sessions. More studies, with adequate one-to-one control conditions, should be conducted to clarify this aspect.

\section{Effect on Mood}

The present study showed an improvement in the CSDD mood scores during the intervention in MSSE and activity groups, although this improvement was not significant. However, for both groups, the scores worsened during the follow-up period compared to the posttrial assessment. One explanation is that the positive effect on the patients' mood is determined by the one-to-one attention rather than the multisensory stimulation. ${ }^{14}$ According to this theory, oneto- one therapy, either MSSE or activity sessions, could prevent the worsening of depressive symptomatology in people with dementia. Previous studies that compared an MSSE group with a control group that received one-to-one therapy did not observe significant differences in the mood of the 2 groups in the short term ${ }^{11,14}$ or long term. ${ }^{15}$

Positive aspects of one-to-one therapies, as MSSE or activity sessions, can be attributed to the higher time and effort being spent with the person with dementia, the perceived qualitative shift in the relationship between the patient and the staff and as a result, elderly care improvement. O'Connor et al ${ }^{35}$ provided a systematic review, the use of psychosocial treatments in people with dementia, concluding that the benefits cannot be attributed confidently to a unique therapeutic modality, and in some instances, benefits in the symptoms can be attributed to the empathic human interaction with the staff. MSSE, like other one-to-one interventions, may be a useful method of managing the psychological symptoms of dementia; however, as there is currently limited evidence to support the efficacy of MSSE, in most instances, the efficacy of using these for residents needs to be determined on an individual basis. ${ }^{36}$ 


\section{Effect on Cognitive Level}

Concerning cognitive level, we found no significant effects following MSSE or significant differences with the activity and control groups.

The effects of multisensory stimulation on the cognitive status of elderly people with dementia have been hardly studied. In consonance with our results, in people with moderate to severe dementia, Baker et $\mathrm{al}^{15}$ did not observe significant effects of the MSSE on the cognitive level or between the MSSE group and the group that received one-to-one activity sessions. However, Ozdemir and Akdemir, ${ }^{37}$ in the case of

mildly affected patients with Alzheimer's disease, found a significant improvement in the MMSE scores $(\mathrm{P}=.001)$ after a group multisensory intervention that included groups of 4 or 5 people and a combination of musical therapy, painting, and sensory stimulation. Furthermore, this positive effect remained 3 weeks after the intervention was completed. However, these results must be interpreted with caution because the study lacked a control group and, therefore, the results could not be compared.

Consequently, MSSE could have a positive efficacy for managing the cognitive impairment of people with early dementia. Nevertheless, there is no evidence to support its efficacy in people with moderate or severe dementia.

\section{Effect on Functional Status in ADL}

Previous studies have suggested that MSSE sessions have short-term positive effects on the functional status of people with dementia. ${ }^{38,39}$ However, the current study found no long-term benefits for functional status based on the Barthel ADL score or significant differences between the MSSE group and the other 2 groups. A previous research study on the long term effects of MSSE on the balance of institutionalized people with dementia17 did not find positive effects after a period of 6 weeks of intervention or significant differences compared to the control group, which received individual visits from volunteers.

\section{Limitations and Recommendations for Future Research}

One limitation from the present study is the small sample size included in each group. The small samples may account for the nonsignificant results found in some of the outcome measures. Future empirical studies with larger samples are necessary to confirm our results.

Another aspect to take into account is that the Barthel test really did not assess the impact of the intervention in the functional status, being only measured in terms of ADL. Besides, the findings for the cognitive effect assessed by the MMSE are limited in people with high cognitive impairment since MMSE shows "floor" levels when patients progress to moderate or severe dementia stages. ${ }^{40,41}$ A possible hypothesis is that people in advanced stages, with very low level of functioning, may benefit more from MSSE than from more cognitively demanding one-to-one activities. ${ }^{6,15}$ Therefore, future research should conduct specific studies with people in advanced stages of dementia, using tools that allow more specific discriminations of the variations in the cognitive and functional status in ADL for these people.

The MSSE using an MSSR implies economic resources higher than those required in other one-to-one therapies for people with dementia. Therefore, it is especially necessary to demonstrate in an empirical way that its benefits on symptoms of people with dementia are better than those provided by one-to-one attention. Resources such as the manpower and the costs of setting up an MSSE cannot be justified without such evidence. $^{42}$

\section{Conclusions}

The results of this study show that MSSE can have long-term positive effects on some of the neuropsychiatric symptoms of institutionalized people with dementia. Patients treated with MSSE had a significantly higher improvement in physically nonaggressive behavior than those who attended one-toone activity sessions. In both the MSSE group and the activity group, there was a significant improvement in the total CMAI, verbally agitated behavior, and total NPI-NH scores in pre-, mid-, and postintervention.

Future empirical studies with adequate designs and larger samples are necessary to confirm the MSSE's long-term effectiveness on agitated behavior, independently of the effect of a one-to-one intervention. 


\section{Acknowledgments}

We thank the users and staff of the Gerontology Complex La Milagrosa, without whom the study would not have been possible. We are truly grateful to Prof Roger Baker for providing us information to design a randomized control trial of the Snoezelen multisensory environment for patients with dementia.

\section{Declaration of Conflicting Interests}

The authors declared no potential conflicts of interest with respect to the research, authorship, and/or publication of this article.

\section{Funding}

The authors received no financial support for the research, authorship, and/or publication of this article.

\section{References}

1. Burns I, Cox H, Plant H. Leisure or therapeutics? Snoezelen and the care of older persons with dementia. Int J Nurs Pract. 2000; 6(3):118-126

2. Moffat N, Barker P, Pinkney L. Snoezelen: An Experience for People With Dementia. Chesterfield: Rompa; 1993.

3. Milev RV, Kellar T, McLean M, et al. Multisensory stimulation for elderly with dementia: a 24-week single-blind randomized controlled pilot study. Am J Alzheimers Dis Other Demen. 2008; 23(4):372-376.

4. Kovach CR. Sensoristasis and imbalance in persons with dementia. J Nurs Scholarsh. 2000;32(4):379-384

5. Chitsey AM, Haight BK, Jones MM. Snoezelen: a multisensory environmental intervention. J Gerontol Nurs. 2002;28(3):41-49.

6. Baker R, Bell S, Baker E, et al. A randomized controlled trial of the effects of multi-sensory stimulation (MSS) for people with dementia. Br J Clin Psychol. 2001;40(pt 1):81-96.

7. Finnema E, Drös RM, Ribbe M, Van Tilburg W. The effects of emotion-oriented approaches in the care for persons suffering from dementia: a review of the literature. Int J Geriatr Psychiatry. 2000;15(2):141-161

8. Herrmann N, Gauthier S. Diagnosis and treatment of dementia: 6. Management of severe Alzheimer disease. CMAJ. 2008;179(12): 1279-1287

9. Sánchez A, Millán-Calenti JC, Lorenzo-López L, Maseda A. Multisensory stimulation for people with dementia: a review of the literature. Am J Alzheimers Dis Other Demen. 2013:28(1):7-14.

10. Cornell A. Evaluating the effectiveness of Snoezelen on women who have a dementing illness. Int J Psychiatr Nurs Res. 2004; 9(2):1045-1062.

11. Baillon S, Van Diepen E, Prettyman R, Redman J, Rooke N, Campbell R. A comparison of the effects of Snoezelen and reminiscence therapy on the agitated behaviour of patients with dementia. Int J Geriatr Psychiatry. 2004;19(11):1047-1052.

12. Minner D, Hoffstetter P, Casey L, Jones D. Snoezelen activity: the good shepherd nursing home experience. J Nurs Care Qual. 2004; 19(4):343-348

13. Hope KW. The effects of multisensory environments on older people with dementia. J Psychiatr Ment Health Nurs. 1998; 5(5):377-385

14. Cox H, Burns I, Savage S. Multisensory environments for leisure: promoting well-being in nursing home residents with dementia. J Gerontol Nurs. 2004;30(2):37-45

15. Baker R, Holloway J, Holtkamp CC, et al. Effects of multisensory stimulation for people with dementia. J Adv Nurs. 2003;43(5):465-477.

16. Ward-Smith P, Llanque SM, Curran D. The effect of multisensory stimulation on persons residing in an extended care facility. Am J Alzheimers Dis Other Demen. 2009;24(6):450-455.

17. Klages K, Zecevic A, Orange JB, Hobson S. Potential of Snoezelen room multisensory stimulation to improve balance in individuals with dementia: a feasibility randomized controlled trial. Clin Rehabil. 2011;25(7):607-616.

18. Baker R, Bell S, Assey J, et al. A Randomized Controlled Trial of the Snoezelen Multi-Sensory Environment for Patients With Dementia. Dorset, United Kingdom: Research and Development Support Unit, Poole Hospital; 1998.

19. Pace GM, Ivancic MT, Edwards GL, Iwata BA, Page TJ. Assessment of stimulus preference and reinforcer value with profoundly retarded individuals. J Appl Behav Anal. 1985;18(3):249-255.

20. Cervilla JA, Rodríguez Cano T, Gurpegui M. Prevalencia de Conductas Agitadas en Ancianos. An Psiquiatr. 1995;11(suppl I):5-6.

21. Cohen-Mansfield J, Marx MS, Rosenthal AS. A description of agitation in a nursing home. J Gerontol A Biol. 1989;44(3):M77M84.

22. Boada M, Tárraga L, Modinos G, López OL, Cummings JL. Neuropsychiatric inventory-nursing home version (NPI-NH): validación española. Neurologia. 2005;20(10):665-673.

23. Wood S, Cummings JL, Hsu MA, et al. The use of the neuropsychiatric inventory in nursing home residents. Characterization and measurement. Am J Geriatr Psychiatry. 2000; 8(1):75-83.

24. Pujol-Doménech J, de Azpiazu P, Salamero M, Cuevas R. Sintomatología depresiva de la demencia. Escala de Cornell: validación de la versión en castellano. Rev Neurol. 2001; 33(4):397-398.

25. Folstein MF, Folstein SE, McHugh PR. Mini-Mental state: a practical method for grading the cognitive state of patients for the clinician. J Psychiatry Res. 1975;12(3):189-198.

26. Blesa R, Pujol M, Aguilar M, et al; NORMACODEM Group. Clinical validity of the mini-mental state for Spanish speaking communities. Neuropsychologia. 2001;39(11):1150-1157.

27. Reisberg B, Ferris SH, de León MJ, Crook T. Global deterioration scale (GDS). Psychofarmacol Bull. 1988;24(4):661-663.

28. Mahoney FI, Barthel DW. Functional evaluation: the Barthel index. Md State Med J. 1965;14:61-65. 
29. Baztán JJ, Pérez del Molino J, Alarcón T, San Cristóbal E, Izquierdo G, Manzarbeitia J. Índice de Barthel: Instrumento válido para la valoración funcional de pacientes con enfermedad cerebrovascular. Rev Esp Geriatr Gerontol. 1993;28(1): 32-40.

30. SPSS PASW1. Statistics 18 Core System User's Guide. Chicago: SPSS Inc; 2009

31. Lyketsos C, López O, Jones B, Fitzpatrick AL, Breitner J, DeKosky S. Prevalence of neuropsychiatric symptoms in dementia and mild cognitive impairment. Results from the cardiovascular health study. JAMA. 2002;288(12):1475-1483.

32. Howard R, Ballard C, O`Brien J, Burns A. Guidelines for the management of agitation in dementia. Int J Geriatr Psychiatry. 2001; 16(7):714:717.

33. Ballard C, Corbett A. Management of neuropsychiatrie symptoms in people with dementia. CNS Drugs. 2010;24(9):729-739.

34. Lancioni GE, Cuvo AJ, O'Reilly MF. Snoezelen: an overview of research with people with developmental disabilities and dementia. Disabil Rehabil. 2002;24(4):175-184.

35. O'Connor DW, Ames D, Gardner B, King M. Psychosocial treatments of behavior symptoms in dementia: a systematic review of reports meeting quality standards. Int Psychogeriatr. 2009;21(2): 225-240.

36. Bauer M, Rayner JA, Koch S, Chenco C. The use of multi-sensory interventions to manage dementia-related behaviours in the residential aged care setting: a survey of one Australian state. J Clin Nurs. 2012;21(21-22):3061-3069.

37. Ozdemir L, Akdemir N. Effects of multisensory stimulation on cognition, depression and anxiety levels of mildly-affected Alzheimer's patients. J Neurol Sci. 2009;283(1-2):211-213.

38. Staal JA, Sacks A, Matheis R, et al. The effects of Snoezelen (multi-sensory behavior therapy) and psychiatric care on agitation, apathy, and activities of daily living in dementia patients on a short term geriatric psychiatric inpatient unit. Int J Psychiatry Med. 2007;37(4):357-370.

39. Collier L, McPherson K, Ellis-Hill C, Staal J, Bucks R. Multisensory stimulation to improve functional performance in moderate to severe dementia-interim results. Am J Alzheimers Dis Other Demen. 2010;25(8):698-703.

40. PeavyGM, Salmon DP, Rice VA, et al. Neuropsychological assessment of severely demeted elderly: the severe cognitive impairment profile. Alzheimer Dis Assoc Disord. 2000;14(3):168-175.

41. Harrell LE, Marson D, Chatterjee A, Parrish JA. The severe mini-mental state examination: a new neuropsychologic instrument for the bedside assessment of severely impaired patients with Alzheimer disease. Alzheimer Dis Assoc Disord. 2000; 14(3):168-175.

42. Chung JCC, Lai CKY. Snoezelen for dementia. Cochrane Database Syst Rev. 2009;(4):CD003152. 